\title{
ISOLAMENTO E SELEÇÃO DE FUNGOS PARA CONTROLE DE LARVAS DE TERCEIRO INSTAR DE MUSCA DOMESTICA
}

\section{E.G. Fernandes ${ }^{1}$, L.D.S. Durães ${ }^{2}$, M.A.Z. Borges $^{2}$, H.M. Valério ${ }^{2}$}

${ }^{1}$ Universidade Federal do Rio Grande do Sul, Instituto de Microbiologia, Rua Sarmento Leite, 500, CEP 90050170, Porto Alegre, RS, Brasil. E-mail: eliogomesfernandes@hotmail.com

\section{RESUMO}

O controle biológico no Brasil vem crescendo devido aos problemas gerados pelo uso indiscriminado de inseticidas químicos. A Musca domestica representa o maior problema em granjas avícolas devido às condições favoráveis para seu crescimento populacional. Sendo assim, foram realizadas capturas de dípteros muscoides em um aviário na região de Montes Claros, MG, usando armadilhas contendo isca química e captura por busca direta e, destas moscas, foram isolados e identificados fungos residentes nestes insetos. Os fungos isolados foram duas espécies do gênero Aspergillus sp., um do gênero Memnoniella sp., Scopulariopsis sp., Paecilomyces sp e um fungo da família Moniliaceae. Foram também requeridos junto ao CENARGEM/EMBRAPA as espécies de fungos entomopatogênicos Beauveria bassiana CG 470 e CG 472; Metarhizium anisopliae CG 34 e CG 312 e o Paecilomyces sp. CG 301. As espécies selecionadas para os bioensaios foram um Aspergillus sp., Memnoniella sp. e os Metarhizium anisopliae CG 34 eCG 312 por terem boa esporulação. Os fungos Aspergillus sp. e o Memnoniella sp. não apresentaram capacidade entomopatogênica, os fungos M. anisopliae CG 34 e CG 312 foram bastante eficientes em controlar a emergência dos adultos de $M$. domestica, mostrando-se bons agentes de controle biológico.

PALAVRAS-CHAVE: Fungos entomopatogênicos, bioensaios e controle biológico.

\section{ABSTRACT}

ISOLATION AND SELECTION OF FUNGI IN ORDER TO CONTROL MUSCA DOMESTICA THIRD-INSTAR LARVAE. Use of biological control measures in Brazil has been increasing because of the problems generated from the indiscriminate use of chemical insecticides. Musca domestica represents the biggest problem to poultry farms due to the favorable conditions for its population growth. The present study was therefore conducted, beginning with captures of muscoid diptera on a poultry farm in the Montes Claros region, state of Minas Gerais, Brazil, using traps containing chemical bait and as well as capture by direct search, and the fungi resident on these flies was isolated and identified. The isolated fungi were two species of Aspergillus sp., one each from the genera Memnoniella sp., Scopulariopsis sp., Paecilomyces sp., and one fungi of the family Moniliaceae. Also, the entomopathogenic species of fungi Beauveria bassiana CG 470 and CG 472, Metarhizium anisopliae CG 34 and CG 312, and Paecilomyces sp. CG 301 were requested from CENARGEM/EMBRAPA. The species selected for the bioassays were Aspergillus sp., Memnoniella sp. and Metarhizium anisopliae CG34 and CG 312. The fungi Aspergillus sp. and the Memnoniella sp. did not present entomopathogenic capacity; the fungi Metarhizium anisopliae CG 34 and CG 312 were sufficiently efficient in controlling the emergence of the adults of Musca domestica, showing themselves good agents of biological control.

KEY WORDS: Entomopathogenic fungi, biossay, biological control.

\section{INTRODUÇÃO}

Os dípteros muscoides são considerados o principal problema em granjas de galinha de postura, por seu grande potencial biótico neste ambiente e pelos danos diretos e indiretos causados pela presença de altas densidades destes dípteros em gran- jas. (Axtell; Arends, 1990). Estas moscas produzidas no esterco da granja podem migrar e colonizar cidades e vilarejos próximos dos empreendimentos, causando um problema de saúde pública (WINPISINGER, 2005). Perante a situação, a população atingida pode acionar o serviço de saúde local e exigir até o fechamento da granja.

${ }^{2}$ Universidade Estadual de Montes Claros, Departamento de Ciências Biológicas e da Saúde, Montes Claros, MG, Brasil. 
BicHO et al. (2004), em coleta feita em um aviário, constataram que $61,47 \%$ dos artrópodes existentes no localcorrespondiama dípteros. Aalta densidadedeaves e o acúmulo de esterco servem de substrato ideal para a proliferaçãodemoscas, destacando-sea Muscadomestica que é veiculadora de micro-organismos que causam diversas patologias tanto em pessoas que trabalham ou moramnasredondezasetambémcausamsériasdoenças nas aves (COSTA et al., 2004; BERNARDi et al., 2006).

O controle das populações de moscas é altamente desejável, devido aos danos que causame, na maioria das vezes, é conseguido através do manejo químico, com a aplicação de inseticidas. A utilização dos inseticidas de maneira indiscriminada ou não-estratégica faz com que muitas espécies de insetos desenvolvam resistência. A $M$. domestica é uma das espécies com maior capacidade de desenvolver resistência aos inseticidas, e os fatores que influenciam o desenvolvimento desta resistência são genéticos, biológicos e operacionais. O surgimento da resistência faz com que seja necessário o desenvolvimento de novas classes de pesticidas, o que aumenta o preço final do controle químico (KeIDING, 1999).

Como alternativa ao controle químico, o controle microbiano de insetos utilizando fungos entomopatogênicos vem sendo aplicado utilizando-se principalmenteas espécies Metarhiziumanisopliaee Beauveria bassiana. Entretanto, poucos são os estudos direcionados ao controle biológico de moscas por fungos entomopatogênicos, avaliando a ação destes sobre as diversas formas evolutivas destes dípteros (BERNARDi et al., 2006; NunEs et al., 2002).

Devido aos patógenos transmitidos por esses insetos-pragas, o controle microbiano usando fungos entomopatogênicos é uma alternativa viável esegura, pois são considerados seguros tanto para os animais como para o ambiente e, além disso, a permanência desses micro-organismos no ambiente é potencialmente maior do que a dos produtos químicos (ALVES, 1998; AlEXANDRE et al., 2006).

Oobjetivodestetrabalhofoi isolar fungos residentes em $M$. domestica e, por meio de bioensaios, avaliar a competência de cada isolado em controlar larvas de $3^{\circ}$ instar de $M$. domestica em laboratórioecompará-loscom a ação de fungos entomopatogênicos de uso comercial.

\section{MATERIAISEMÉTODOS}

Os experimentos de campo foram realizados em dois galpões em uma granja aviária, localizada na BR 365, km14, no Município de Montes Claros, MG. As coletas foram realizadas de fevereiro a maio de 2007, sendo realizadas coletas semanais, utilizando armadilhas feitas com garrafas plásticas do tipo PET dedois litros, furadas perto da base para entrada das moscas e pintadas de amarelo para aumentar a atratividade (BURG; AxTELL, 1984). Como isca atrativa, foi usada a isca comercial Vetor ${ }^{\circledR}$ (Novartis Biociências). Foram colocadas quatro armadilhas em cada galpão na granja. As coletas foram feitas também utilizando um puçá de tela de Nylon para captura de indivíduos vivos de $M$. domestica, e essas moscas capturadas foram criadas no Laboratório de Zoologia da UNIMONTES.

Os adultos vivos de $M$. domestica coletados no aviário foram levados ao Laboratório de Zoologia e criados em caixa entomológica de acrílico a temperatura ambiente. Dentro da caixa entomológica foi mantida uma placa de Petri com algodão eágua para manter a umidade. A alimentação dos adultos ocorreu com leite em pó adicionado de açúcar ad libidum e para obtenção das larvas foi utilizado um meio de cultura composto de ração avícola de postura mais farelo de trigo na proporção de $50 \%$ cada.

As amostras coletadas no aviário foram individualizadas em sacos plásticos e identificadas no Laboratório de Biotecnologia da UNIMONTES e transferidas para bandejas plásticas, sendo coletados adultos vivos ou mortos de $M$. domestica. Todos os insetos passaram por desinfecção por imersão em álcool $70 \%$ por 2 segundos e, em seguida, imersos em hipoclorito $1 \%$ por 1 minuto, posteriormente repetindo-se a lavagem em água destilada estéril para a retirada do excesso de cloro.

Concluído o processo de desinfecção, os insetos foram transferidos para câmara úmida, com 25 insetos por placa, totalizando 450 moscas. Todos os indivíduos foram mantidos a $26 \pm 1^{\circ} \mathrm{C}$ e fotofase de 14 h por 27 dias para obter o crescimento dos fungos residentes.

Os fungos foram isolados dos cadáveres, transferindo-os para placas contendo meio de cultura Batata Dextrose Agar, acrescido de tetraciclina (na proporção de $100 \mathrm{mg}$ de tetraciclina para 0,1 L de BDA). Posteriormente, os fungos foram identificados por meio de microcultivo e chave taxonômica proposta por Alves (1998) e BARNETT; HUNTER (1998), e armazenados em tubos de ensaio com meio BDA, recobertos por óleo mineral estéril e usados posteriormente nos bioensaios (Alves, 1998).

Foram gentilmente cedidos pelo CENARGEN Embrapa Recursos Genéticos, Brasília, DF, os seguintes fungos entomopatogênicos: B. bassiana CG470eCG 472; M. anisopliae var. anisopliae CG 34 e CG 312; Paecilomyces sp. CG301; Trichoderma harzianum CG502.

As larvas das moscas foram obtidas da criação do Laboratório deZoologia da UNIMONTES, a partir da F2 (2 ${ }^{\text {a }}$ geração) etodas estavam sadias ebem nutridas, sendo selecionadas as larvas com 72 horas de eclosão. Por meio desses bioensaios, foram analisados os diferentes níveis de respostas dos hospedeiros tratados. As larvas tiveram a mesma idade, e fase de desenvolvimento biológico e o número mínimo para cada 
tratamento foi de 60 indivíduos. Os fungos selecionados para os bioensaios foram os que esporularam em meio de cultura BDA ou BDA com adição de caldo obtido com fervura de arroz.

Os fungos que não esporularam, foram transferidos para meio de cultura BDA modificado. A alteração do meio de cultura ocorreu acrescentando água retirada da fervura de arroz integral em substituição a água destilada, melhorando assim o valor nutricional do meio de cultura.

As suspensões de conídios foram preparadas acrescentando $6 \mathrm{~mL}$ de água destilada estéril adicionado de espalhante adesivo (Tween 80 ) na proporção de $0,01 \%$, sobre a superfície de cada placa contendo as culturas esporuladas. Em seguida, foi feita a raspagem superficial para a retirada dos conídios e essas suspensões de esporos foram então transferidas para tubos de ensaio, para posterior homogeneização e quantificação, em câmara de Neubauer.

Após a obtenção das larvas de $M$. domestica, estas foram colocadas em uma placa de Petri, sendo 60 larvas para cada tratamento com diferentes diluições de esporos. As suspensões de esporos foram então despejadas nas placas sobre as larvas de $M$. domestica, transferindo-se, em seguida, essas larvas para potes plásticos de $280 \mathrm{~mL}$, previamente esterilizados por radiação ultravioleta, contendo chumaços de algodão estéril, umedecidos com água destilada estéril, sendo o tratamento controle composto por água destilada estéril adicionada de $0,01 \%$ de Tween 80 . As larvas foram divididas em 3 grupos de 20 indivíduos cada e o tratamento com cada diluição de esporo foi feito em triplicata. Todos os grupos foram mantidos em incubadoras B. O. D com fotofase de 14 h a $27^{\circ} \mathrm{C} \pm 1$. As observações foram feitas diariamente. As larvas não foram alimentadas durante a incubação e este foi avaliado por um período de dez dias, sendo a porcentagem de mortalidade o fator analisado.

Para verificar o efeito da concentração de esporos das quatro espécies de fungo sobre a mortalidade dos indivíduos foi construído eajustado um modelolinear generalizado,com distribuição deerros quasibinomial, por ter sido a mais adequada para o tipo de dados (proporção de indivíduos mortos). As variáveis independentes domodeloforam: concentração deesporos, fungo (com quatroníveis, correspondentes às espécies de fungo) e a interação entre concentração de esporos e fungo (concentração de esporos $x$ fungo). Os valores de concentração de esporos foram linearizados com a função $\log _{10}(x)$, exceto os valores iguais a zero. Foi realizada uma Análise deDesvio(ANODEV) domodelo, que é uma generalização da análise da variância para os Modelos Lineares Generalizados (GLM). Uma AnálisedeContrastes foi realizada para verificar quais fungos são diferentes entre si, quanto aos seus efeitos na mortalidade das larvas. Todas as análises estatísti- cas foram realizadas no software estatístico $\mathrm{R}$ ( $\mathrm{R}$ Core Team, 2008).

\section{RESULTADOSEDISCUSSÃO}

Os fungos isolados e identificados foram pertencentes a duas espécies de Aspergillus sp., Paecilomyces sp., Memnoniella sp., Scopulariopsis sp. e um fungo da família Moniliaceae.

Entre os fungos isolados, apenas os fungos Paecilomyces sp. e o Aspergillus sp. já haviam sido isolados de M.domestica (Alves, 1998; SALEset al., 2002; BANJO et al., 2005), entretanto, não foram encontrados registros do isolamento de Memnoniella sp. e Scopulariopsis sp. em $M$. domestica. O fungo também isolado pertencente à família Moniliceae ainda será identificado para que então, posteriormente à identificação, ele possa ser confirmado ou não como sendo um fungo já isolado de indivíduos de $M$. domestica.

Desses fungos isolados, o gênero Aspergillus pode crescer sobre um grande número de substratos, em virtude da sua capacidade de produção de diferentes tipos de enzimas (CARLILE et al., 2001). Embora em estudos como o de NunEs et al. (2002) e confirmado pelos resultados desse trabalho, espécies do gênero Aspergillus não sejam consideradas entomopatogênicas, esse gênero é relatado como um agente secundário no processo-doença, sendo mais comum sua ocorrência em insetos moribundos, já colonizados por outros patógenos ou submetidos a diferentes tipos de estresse (Alves, 1998).

O gênero Paecilomyces, tal como isolado de $M$. domestica neste trabalho, reúne diversas espécies entomopatogênicas, sendo as mais frequentes $P$. farinosus, $P$. tenuipes, $P$. amoeneroseus, $P$. cicadidae, $P$. fumosoroseus e outros. Esse gênero causa em insetos a doença denominada muscardine amarelo (ALVES, 1998) e, apesar de reunir diversas espécies entomopatogênicas, também são considerados saprófitas no meio ambiente (BARNETT; HunTER, 1998).

O gênero Scopulariopsis, também isolado desses dípteros, apresenta espécies de importância médica. O S.brevicaulis éo patógeno mais comum desse gênero e um dos frequentes contaminantes de laboratórios. Sua importância na medicina é devido ao fato dele causar infecção nas unhas dos pés e, menos comumente, das unhas das mãos (MurRAY, 2002). Dentro desse gênero, existem espécies que podem causar deterioração de laticínios e carnes (FRANCO; LANDGRAF, 2005) sendo também considerado saprófita no solo(BARNETT; HuNTER, 1998). Não foram encontrados registros deisolamento desse gênero em $M$. domestica, e também não foram localizados trabalhos que tenham avaliado se espécies desse gênero apresentam alguma atividade entomopatogênica. 
Para o gênero Memnoniella, não foram encontrados muitos trabalhos, inclusive sobre se possuem potencial entomopatogênico. Como observado nos resultados dos bioensaios, não foi observada atividadeentomopatogênica em indivíduos de $M$. domestica.

Foi isolado um fungo da família Moniliaceae e neste grupo encontram-se espécies que possuem atividade entomopatogênica como Beauveria, Trichorderma e outros não entomopatogênicos como Aspergillus e Penicillium (SILVEIRA, 1995).

Dentre os fungos usados no experimento, apenas os M. anisopliae CG 34 e CG 312 e um dos Aspergillus sp. apresentaram esporulação em meio de cultura BDA, sendo assim, foi realizada uma tentativa de modificar o meio decultura BDA acrescentandoágua retirada da fervura contendo arroz integral e, após esse procedimento, apenas o fungo Memnoniella sp. apresentou esporulação. Devido ao fato dos outros fungosnão terem produzidoesporos em quantidades satisfatórias, os bioensaios foram realizados com esses quatro fungos e cada um obteve uma produção de esporos diferenciada (Tabela 1).

O efeito da concentração de esporos sobre a mortalidadede larvas dependeu da espécie de fungo ( $\mathrm{P}<0,0001$ ) (Tabela 2, Fig. 1). A mortalidade de larvas aumenta com o aumento da concentração de esporos nas duas linhagens de $M$. anisopliae, que não apresentaram efeitos diferentes na mortalidade $(\mathrm{P}<0,0001)$. Aspergillus sp. e Memnoniella sp. não provocaram au- mento na mortalidade das larvas, e também não apresentaram efeitos diferentes na mortalidade (Fig. 1).

Nos bioensaios, verificou-se que o fungo Memnoniella sp. não impediu a pupação das larvas, mostrando-se sem potencial entomopatogênico, sendo que o aumento da concentração de esporos na suspensão aumentou a emergência de adultos (Fig. 1).

O Aspergillus sp. também se mostrou pouco eficiente para o controle de larvas como entomopatógeno (Fig. 1). NunEset al. (2002) observaram emergências de adultos entre $57,50 \%$ e $86,25 \%$ quando as larvas foram tratadas com suspensões de $10^{8}$ esporos $/ \mathrm{mL}$, sendo que estes valores indicam que a porcentagem deemergência de adultos foi inversamente proporcional aos aumentos das concentrações das suspensões de esporos com as duas espécies de Aspergillus sp. avaliadas. O mesmo resultado não foi observado nestes bioensaios, onde não houve diferença entre o aumento da concentração de esporos / $\mathrm{mL}$ e a taxa de mortalidade larval (Tabela 2, Fig. 1).

Os isolados do fungo $M$. anisopliae mostraram-se bastantes eficientes como entomopatógenos de larvas deM. domestica (Fig. 1), sendo esses resultados já observados por BERNARDi et al. (2006) e BARSON et al. (1994). A diferença demortalidadeobservadaentreosisoladosdo gênero Metarhizium poderia ser atribuída à diferença de concentração de esporos $/ \mathrm{mL}$, sendo queo $M$. anisopliae CG 312 teve uma maior esporulação e, consequentemente, seuinóculofoibem mais concentrado(Tabela1).

Tabela 1 - Concentrações de esporos/mL usadas nos bioensaios após recuperação da suspensão micelial no meio de cultura.

\begin{tabular}{lcccr}
\hline Fungo (espécie) & \multicolumn{3}{c}{ Diluições de esporos (esporos/mL) } \\
\cline { 2 - 5 } & $10^{-1}$ & $10^{-2}$ & $10^{-3}$ & $10^{-4}$ \\
\hline Memnoniella sp. & $2,1 \times 10^{5}$ & $2,1 \times 10^{4}$ & $2,1 \times 10^{3}$ & $2,1 \times 10^{2}$ \\
Aspergillus sp. & $7,2 \times 10^{6}$ & $7,2 \times 10^{5}$ & $7,2 \times 10^{4}$ & $7,2 \times 10^{3}$ \\
Metarhizium anisopliae CG 34 & $5,8 \times 10^{6}$ & $5,8 \times 10^{5}$ & $5,8 \times 10^{4}$ & $5,8 \times 10^{3}$ \\
Metarhizium anisopliae CG 313 & $8,2 \times 10^{6}$ & $8,2 \times 10^{5}$ & $8,2 \times 10^{4}$ & $8,2 \times 10^{3}$ \\
\hline
\end{tabular}

Tabela 2 - Resultado da análise de desvio do modelo linear generalizado que explica a mortalidade de larvas. Todas as variáveis independentes tiveram um efeito significativo sobre a mortalidade.

\begin{tabular}{lccccc}
\hline Variáveis & GL $^{\mathrm{a}}$ & GL restante & Desvio $^{\mathrm{b}}$ & Desvio residual & $P$ \\
\hline Modelo nulo & - & 50 & - & 406,97 & - \\
Log (concentração de esporos) & 1 & 49 & 61,45 & 345,52 & $<0,0001$ \\
Fungo & 2 & 47 & 125,55 & 219,97 & $<0,0001$ \\
Log (concentração de esporos): Fungo & 2 & 45 & 105,85 & 114,12 & $<0,0001$ \\
\hline
\end{tabular}

${ }^{a} \mathrm{GL}$ : Graus de liberdade.

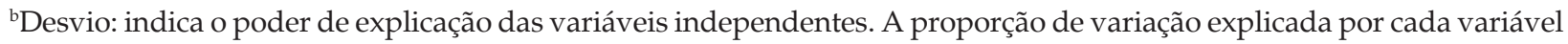
independente é calculada dividindo o desvio de cada variável pelo desvio total $(406,97)$.

A existência da interação (Fungo: concentração de esporos) indica que o efeito da concentração sobre a mortalidade depende da espécie de fungo. 


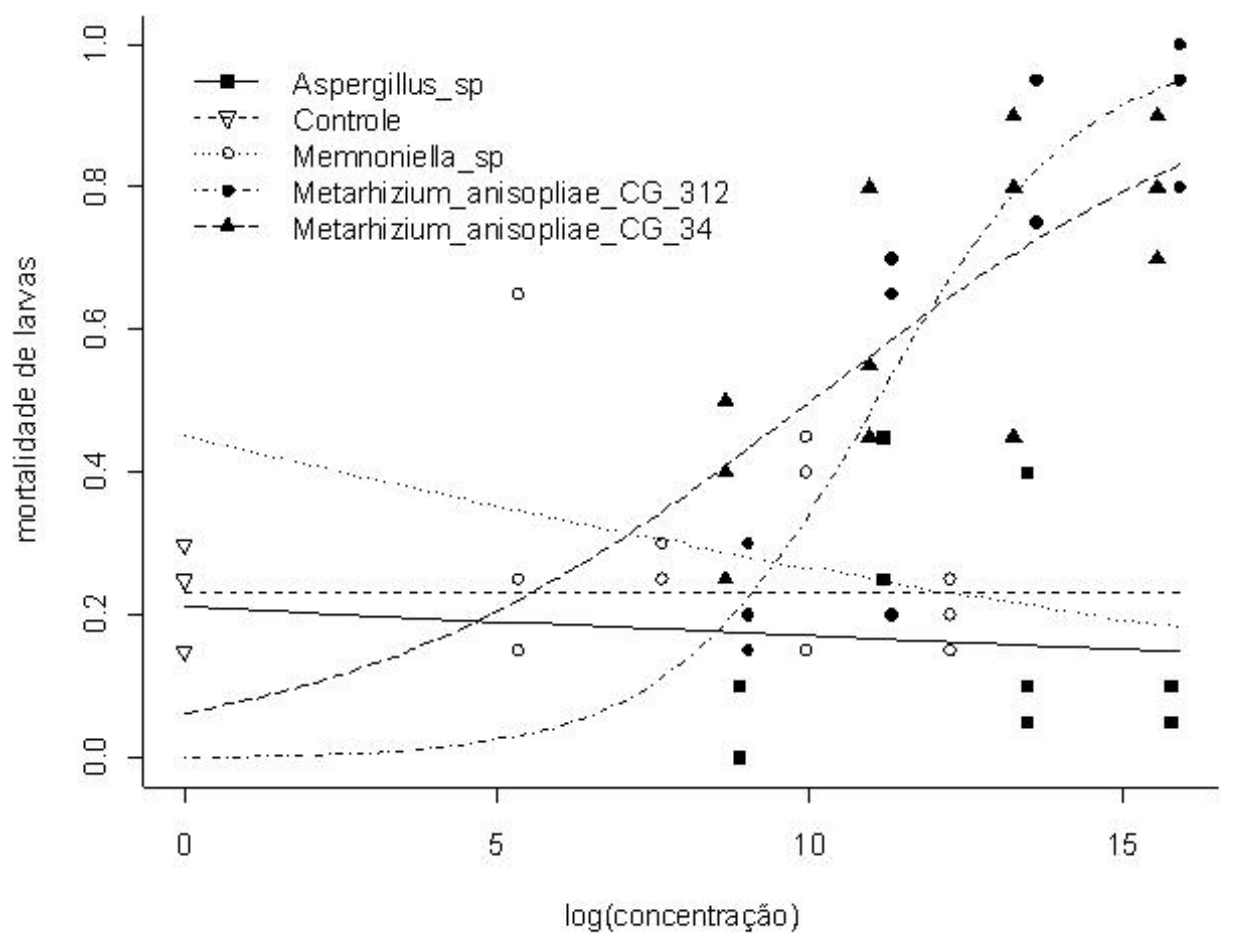

Fig. 1 - Porcentagem de larvas mortas após tratamento com diferentes concentrações de esporos/mL de cada espécie fúngica analisadas neste experimento. Os valores $0,5,10$ e 15 no eixo x significam respectivamente as diluições $10^{-4}$, $10^{-3}$, $10^{-2}$ e $10^{-1}$.

Por meio desses resultados, pôde ser observada a diferença quando larvas de $M$. domestica foram tratadas comfungos entomopatogênicos e comfungos que são saprófitas no ambiente (não entomopatogênico). Os fungos entomopatogênicos como o M. anisopliae têm a capacidade de produzir proteases, esterases, lipases, quitinases e outras enzimas que degradam a cutícula dos insetos e acabam levando a morte do hospedeiro pela invasão e colonização de seus corpos (Tiago; Furlaneto, 2003).

O controle de $M$. domestica em locais de criação de animais vem crescendo a cada ano ejá existem alguns trabalhos onde o controle dessas moscas em aviários, através de fungos entomopatogênicos, foi satisfatório (Kaufman et al., 2005; LuIs; José, 2006). O controle populacional de $M$. domestica usando fungos entomopatogênicos é muito promissor eainda carente de pesquisas, mas vem ganhando bastante atenção principalmente pelo rápido surgimento de resistência aos inseticidas químicos que essas moscas desenvolvem, sendo o controle microbiano uma alternativa viável e segura.

\section{CONCLUSÕES}

Os fungos Scopulariopsis sp. e Memnoniella sp. foram isolados de $M$. domestica, sendo este trabalho o primeiro registro de sua ocorrência.
O gênero Scopulariopsis sp. apresenta espécies de importância médica e veterinária, sendo os adultos de M. domestica veiculadores desses fungos.

Os fungos Memnoniella sp. e Aspergillus sp. isolados das moscas capturadas não apresentaram capacidade entomopatogênica e não são indicados para controle de larvas de $M$. domestica.

Ofungo M. anisopliae (isolados CG34 eCG312) foi bastante eficiente no controle in vitro de larvas de $M$. domestica.

M. anisopliae apresenta maior patogenicidade para larvas de $3^{\circ}$ instar de $M$. domestica quanto maior for a produção de esporos.

\section{AGRADECIMENTOS}

À SOMAI NORDESTE S/A pelo apoio no desenvolvimento da pesquisa, à FAPEMIG pela concessão da bolsa de iniciação cientifica para o primeiro autor durante a sua graduação.

\section{REFERÊNCIAS}

ALEXANDRE, T.M.; ALVES, L.F.A.; NEVES, P.M.O.J.; ALVES, S.B. Efeito da temperatura e cama do aviário na virulência de Beauveria bassiana (Bals.) Vuill. e Metarhizium anisopliae (Metsch.) para o controle do 
cascudinho (Alphitobius diaperinus) (Panzer) (Coleoptera: Tenebrionidae). Neotropical Entomology, v.35, n.1, 2006.

ALVES, S.B. Controle microbiano de insetos. 2.ed. São Paulo: Fealq, 1998. 1163p.

AXTELL, R.C.; ARENDS, J.J. Ecology and management of arthropod pests of poultry. Annual Review of Entomology, v.35, p.101-126, 1990.

BANJO, A.D.; LAEAL, O.A.; ADEDUJI, O.O. Bacteria and fungi isolated from housefly (Musca domestica L.) larvae. African Journal of Biotechnology, v.4, n.8, p.708704, 2005.

BARNETT, H.L.; HUNTER, B.B. Illustrated Genera of imperfect fungi. 4.ed. Minnesota: Burgués Publishing Company, 1998. 218p.

BARSON, G.; RENN, N.; BYWATER, A.F. Laboratoy evaluation of six species of entomopathogenic fungi for the control of the house fly (Musca domestica L.), a pest of intensive animal units. Journal of Invertebrate Pathology, n.64, p.107-113, 1994.

BERNARDI, E.; PINTO, D. M.; NASCIMENTO, J. S. do; RIBEIRO, P.B.; SILVA, C.I. da. Efeito dos fungos entomopatogênicos Metarhizium anisopliae e Beauveria bassiana sobre o desenvolvimento de Musca domestica L. (Díptera: Muscidae) em laboratório. Arquivos do Instituto Biológico, São Paulo, v.73, n.1, p.127-129, 2006.

BICHO, C.L.; ALMEIDA, L.M. de; RIBEIRO, P.B.; SILVEIRA JÚNIOR, P. Flutuação de díptera em granja avícola, Pelotas, Rio Grande do Sul, Brasil. Iheringia. Série Zôologia, v.94, n.2, p.205-210, 2004.

BURG, J.C.; AXTELL, R.C. Monitoring house fly, Musca domestica (Diptera: Muscidae) in caged-layer poultry houses using a baited jug-trap. Environmental Entomology, v.13, n.4, p.1083-1090, 1984.

CARLILE, M.J.; WATKINSON, S.C.; GOODAY, G.W . The fungi. 2.ed. London: Academic Press, 2001. 608p.

COSTA, V.A., FILHO, E.B., NETO, S.S. Parasitóides (Hymenoptera: Chalcidoidea) de moscas sinantrópicas (Díptera: Muscidae) em aviários de Echaporã, SP. Arquivos do Instituto Biológico, São Paulo, v.71, n.2, p.203209, 2004.

FRANCO, B.D.G.M.; LANDGRAF, M. Microbiologia dos Alimentos. São Paulo: Atheneu, 2005.
KAUFMAN, P.E.; REASON, C.; RUTZ, D.A. KETZS, J.K.; ARENDS, J.J. Evaluation of Beauveria bassiana applications against adult house fly, Musca domestica, in commercial caged-layer poultry facilities in New York state. Biological Control, v.33, p.360-367, 2005.

KEIDING, J. Review of the global status and recent development of insecticide resistance in field populations of the housefly, Musca domestica (Diptera: Muscidae). Bulletin of Entomological Research, v.89, 67p. 1999. Supplement 1.

LUIS, J.C.O.; JOSÉ, V.S.D. Control temporal de moscas caseras (Musca domestica) en galpones avícolas mediante nebulizaciones com conídias de Beauveria bassiana. Boletin de Malariologia y Salud Ambiental, v.66, n.2, 2006.

MURRAY, P.R. Microbiologia clínica. 2.ed. Belo Horizonte: MEDSI, 2002. 392p.

NUNES, M.S.; COSTA, G.L. da; BITTENCOUNT, V.R.E.P.; SOUZA, E.J. Avaliação in vitro dos fungos Aspergillus flavus e Penicillium corylophilum em larvas de Musca domestica (Díptera: Muscidae). Parasitologia Latinoamericana, v.57, n.3/4, p.134-140, 2002.

R DEVELOPMENT CORE TEAM. R: A language and environment for statistical computing. Vienna: Foundation for Statistical Computing, 2008.

SALES, M.S.N.; COSTA, G.L.; BITTENCOURT, V.R.E.P. Isolation of fungi in Musca domestica Linnaeus, 1758 (Diptera: Muscidae) captured at two natural breeding grounds in the municipality of Seropédica, Rio de Janeiro, Brazil. Memórias do Instituto Oswaldo Cruz, Rio de Janeiro, v.97, n.8, 2002.

SILVEIRA, V.D. Micologia. 5.ed. Rio de Janeiro: Âmbito Cultural Edições, 1995. 332p.

TIAGO, P.V., FURLANETO, M.C. O papel de proteases degradadoras de cutícula produzidas por fungos entomopatogênicos. Revista do Programa de Ciências Agro-ambientais, v.2, n.1, p.40-51, 2003.

WINPISINGER, K.A; FERKETICH, A.K; BERRY, R.L.; MOESCHBERGER. M.L. Spread of Musca domestica (Diptera: Muscidae), from two caged-layer facilities to neighboring residences in rural Ohio. Journal of Medical Entomology, v.42, n.5, p.732-738, 2005.

Recebido em 5/8/08

Aceito em 5/6/10 